\title{
Effects of antidepressant and treadmill gait training on recovery from spinal cord injury in rats
}

\author{
AF Cristante, TEPB Filho, RP Oliveira, RM Marcon, R Ferreira and GB Santos
}

Study design: Experimental, controlled, animal study.

Objectives: To evaluate the influences of antidepressant treatment, treadmill gait training and a combination of these therapies in rats with experimental, acute spinal cord injury (SCI).

Setting: Brazil.

Methods: 48 Wistar rats were given standardized $\mathrm{SCl}$; rats were then randomly assigned to four treatment groups: (1) motor rehabilitation therapy for 1 hour daily (gait training); (2) daily treatment with the antidepressant, fluoxetine $(0.3 \mathrm{ml}$ per $100 \mathrm{~g}$ intraperitoneally), beginning $24 \mathrm{~h}$ after the trauma; (3) combined fluoxetine treatment and gait training, or (4) untreated (controls). Neurological recovery was tested with the Basso, Beattie and Bresnahan (BBB) scale at 2, 7, 14, 21, 28,35 and 42 days after injury. Moreover, on day 42, all rats underwent a motor-evoked potential test (MEP); then, after euthanasia, histopathological evaluation was conducted in the area of $\mathrm{SCl}$.

Results: Based on the BBB scale, the combined treatment group showed significantly greater improvement compared with the other three groups, from the 14 th to the 42 nd day of observation. The MEP revealed that all treated groups showed significant improvement compared with the control group $(P<0.02$ for latency and $P<0.01$ for amplitude).

Conclusion: Our results indicated that a combination of antidepressant and treadmill gait training was superior to either treatment alone for improving functional deficits in rats with experimental, acute SCl.

Spinal Cord (2013) 51, 501-507; doi:10.1038/sc.2013.18; published online 9 April 2013

Keywords: spinal cord injuries; rehabilitation; central nervous system; injuries; rats

\section{INTRODUCTION}

Despite decades of research, no treatment for spinal cord injury (SCI) has offered complete neurological or functional recovery. ${ }^{1}$ The majority of research has sought solutions in four treatment areas; surgery, biological therapy, physical therapy and medication.

Several recent studies have shown benefit from exercise in animals subjected to SCI. Treadmill training led to improved recovery, coordination and neurological performance. ${ }^{2,3}$ However, no study has evaluated the benefits of exercising in combination with other therapeutic modalities.

Corticosteroids and gangliosides are currently used in humans. ${ }^{4}$ Other drugs are also being tested for the treatment of SCIs, including calcium channel blockers, naloxone, gangliosides, lazaroids, dimethyl sulfoxide and alpha methyl para-tyrosine; but these are mainly administered to prevent secondary damage. ${ }^{5}$ In contrast, a new line of research has shown promising results with antidepressants.

Antidepressants are commonly used to treat depressive symptoms experienced by individuals with SCIs. Moreover, serotonin reuptake inhibitors appear to have a positive effect on limb movement in individuals with SCIs. ${ }^{6-8}$ The loss of regulatory descending serotonergic mechanisms after SCI contributes to motor deficits and causes increased numbers of serotonin receptors in spinal cord segments below the injury. ${ }^{9}$ This increased number of receptors appears to have potential for treatment, because serotonin receptor agonists have been shown to improve motor deficits. ${ }^{6,7}$ Serotonin precursors have also exhibited positive effects on motor recovery. ${ }^{9}$

Experimental studies have sought to elucidate potential synergism among different pharmacological treatments for SCI. For example, serotonergic agonists combined with epidural stimulation provided additive benefits. ${ }^{10}$ We hypothesized that treatment with serotonin reuptake inhibitors combined with treadmill exercise may have additive or synergistic effects on the spinal cord and the hippocampus. This combined treatment has not been clearly studied in SCI models. ${ }^{11}$

This study aimed to evaluate and compare the influences of antidepressant treatment, treadmill gait training and a combination of these treatments on the histology and functional recovery in rats subjected to standardized, experimental SCI.

\section{MATERIALS AND METHODS}

Study design, animals and ethics

This controlled experiment examined functional and histological results of antidepressant therapy and physical training in Wistar rats that had sustained standardized, spinal cord lesions. This study followed all applicable institutional and governmental regulations on the ethical use of animals. The institution's Ethical Committee approved the research protocol.

This study included 48 male Wistar rats (300-340 g), aged 20-21 weeks. All rats were healthy and came from the same university vivarium. All rats were 
submitted to experimental spinal cord lesions, as described below. Rats were then randomly assigned to one of four groups ( $n=12$ animals each), according to numbers drawn from opaque, sealed envelopes. Group 1 received physical therapy, consisting of 1-h treadmill training sessions. Group 2 received daily antidepressant therapy, beginning $24 \mathrm{~h}$ after the trauma. Group 3 received both therapies: physical training and antidepressant. Group 4 received no treatment (control group). Allocation was concealed from the surgeon who performed all laminectomy procedures.

\section{Laminectomy and experimental spinal cord lesion protocol}

Rats were anesthetized with $100 \mathrm{mg} \mathrm{kg}^{-1}$ of ketamine and $5 \mathrm{mg} \mathrm{kg}^{-1}$ of xylazine intraperitoneally; in addition, they were locally anesthetized with lidocaine hydrochloride and epinephrine.

The laminectomy was previously described. ${ }^{12}$ Briefly, a 3-cm long, dorsal, medial incision was performed to expose the spinous process and laminae of vertebrae $\mathrm{T} 9$ and $\mathrm{T} 10$. These vertebrae were removed to expose the spinal cord and to enable the positioning of the tip of the NYU Impactor device (New York University, New York, NY, USA). The impactor was used to create the lesion, ${ }^{13}$ based on a protocol previously described, ${ }^{14}$ where a 10 -g weight was dropped from a height of $12.5 \mathrm{~mm}$. The Impactor device tracked the trajectory of the falling rod ( $\pm 10 \mu \mathrm{m}$ and $\pm 10 \mu \mathrm{s})$ and measured the impact velocity $(\mathrm{Vi})$, cord compression distance $(\mathrm{Cd})$, time $(\mathrm{Ct})$ and compression rate $(\mathrm{Cr}=\mathrm{Cd} / \mathrm{Ct})$. This computerized system ensured that the grade of the injury was standardized (moderate injury). When, for any reason, the impact was greater or less than the intended force $(10 \mathrm{~g}$, at $12.5 \mathrm{~mm}$ height), the animal was excluded.

One author (AFC) performed all surgeries.

After the SCI, rats in groups 1, 2, or 3 received medication and/or physical training. All four groups were evaluated with the Basso, Beattie and Bresnahan (BBB) scale, the evoked potential test, and histopathological analysis of extracted tissues.

\section{Antidepressant therapy}

The pharmacological intervention comprised fluoxetine, delivered intraperitoneally, at a daily dose of $0.3 \mathrm{ml}$ per $100 \mathrm{~g}$ for 42 days, as described previously. ${ }^{11,15}$

\section{Physical training}

One week before the spinal cord lesion, all animals were manipulated, introduced to the training environment, and stimulated to move by placing them on a treadmill at a speed of $6-21 \mathrm{~cm} \mathrm{~s}^{-1}$, for 10 to $15 \mathrm{~min}$. This procedure was performed before the SCI to ensure that they could easily adapt to training after the spinal cord lesion. This preconditioning was implemented to all animals, including the untreated control group. After the SCI, the physical training consisted of $15 \mathrm{~min}$ of treadmill training daily, at a speed of $6-21 \mathrm{~cm} \mathrm{~s}^{-1}, 5$ days per week for 6 weeks.

\section{Functional analysis}

Rats were placed in a $80 \times 80 \mathrm{~cm}$ box, with $17-\mathrm{cm}$ walls, lined with blue cloth. When a rat did not move inside the box, it was stimulated to move after 15$20 \mathrm{~s}$. Two trained observers, blinded to animal allocations and to each other's ratings, used the BBB scale to evaluate the recovery of locomotor capacity ${ }^{16}$ on days $2,7,14,21,28,35$ and 42 after the SCI. In cases of disagreement between observers, the lower value was recorded. Evaluations took 4-5 min each.

\section{Evoked potential test}

On the 42 nd day post-SCI, rats were placed under anesthesia (55-75 mg per kg of intraperitoneal pentobarbital) and subjected to transcranial electrical stimulation to elicit motor-evoked potentials. We recorded the amplitude and latency response in the legs, as described previously. ${ }^{17}$ Briefly, transcranial electrical stimulation at the cortical level elicited neuronal depolarization, which traveled through neural pathways in the spinal cord, and the responses were captured at the muscular level.

\section{Pathological analysis}

After treatment and testing, all animals were euthanized with intravenous thiopental $(65 \mathrm{mg}$ per $100 \mathrm{~g}$ ) and potassium chloride. A $2.5-\mathrm{cm}$ segment of the spinal cord (including the focus of the lesion) was carefully extracted and placed in $4 \%$ paraformaldehyde in saline $(0.1 \mathrm{M})$ for $24 \mathrm{~h}$, and then immersed in $20 \%$ sucrose. The spinal cord was fixed (formalin, 10\%) and three areas were identified: the lesion, the proximal area and the distal area. Each area was sectioned in 2-mm intervals. For hematoxylin-eosin staining, the sections were $5-\mu \mathrm{m}$ thick, cut $1 \mathrm{~cm}$ distally and $1 \mathrm{~cm}$ proximally from the center of the lesion. All fragments were dehydrated in alcohol baths, diaphanized in xylol and impregnated with paraffin. Five-micron thick sections were cut from the paraffin blocks. The slides, previously washed in saline, were then stained with hematoxylin-eosin.

Microscopic evaluations were performed by a single, experienced pathologist (RPO). The sections were classified according to the following variables: necrosis, hemorrhage, hyperemia, axonal degeneration and cellular infiltration. The variables were scored as zero (indicating absence), 1 (discrete), 2 (moderate) and 3 (severe).

The proximal and distal portions of the spinal cord were excised (peripheral, undamaged areas), fixed in osmium tetroxide solution (2\%), and stained with toluidine blue (1\%). Transverse sections of the spinal cord were prepared for electron microscopy $(2-\mu \mathrm{m}$ thick sections, cut sequentially from the center of the lesion to $1 \mathrm{~mm}$ distally and $1 \mathrm{~mm}$ proximally; Figure 1 ). Two visual fields were selected on each section. Each field was photographed at $\times 10000$ for counting fibers to estimate the extent of axon regeneration. Photomicrographs were analyzed with SigmaScan Pro 5.0 software (Systat Software Inc., San Jose, CA, USA). Only neurons with $\geqslant 15 \mu \mathrm{m}$ diameters were counted. ${ }^{18}$ The number of axons in the distal areas was expressed as a percentage of proximal axons according to the following formula: $\mathrm{IR}=$ (number of axons in the distal area/number of axons in the proximal area) $\times 100 .{ }^{19,20}$

\section{Statistical analysis}

Descriptive statistics were presented as means and s.d., with significance set at $P<0.05$. For statistical inference, the data behavior was initially observed, and when outliers were identified, they were submitted to Dixon's $Q$ test for possible deletion. Subsequently, data were tested for normality with the Kolmogorov-Smirnov test. When the distribution was normal, parametric tests, Student's $t$-test and analysis of variance (ANOVA) were employed, as appropriate. The number of factors was adjusted for each situation.

The primary outcome was the BBB index on the 42 nd day. The mean BBB scores were compared with a mixed effects model of two factors: the group $(1,2,3$ and 4) and the week of assessment (1st through 6th). Measures that were repeated throughout the weeks were taken into account. We also evaluated whether an interaction effect existed between these factors. The mixed effects model was adjusted considering covariance matrices that were equal in different groups and unstructured. Initially, the distributions of amplitude and latency measurements from the evoked potential tests were compared among the four study groups with the nonparametric Kruskal-Wallis test. Then, an ANOVA

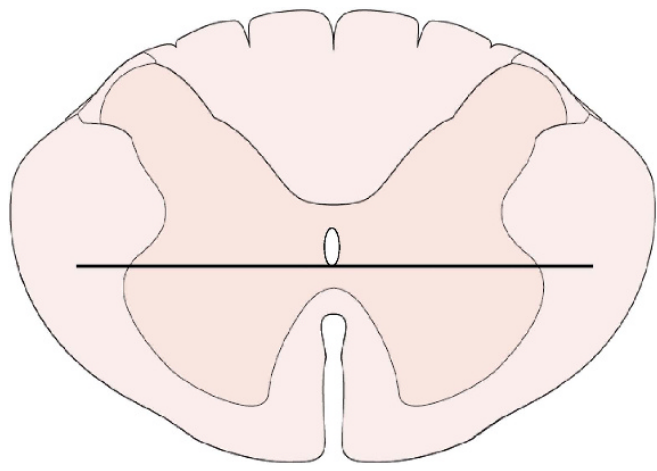

Figure 1 Schematic drawing of the spinal cord with a transverse line in the ventral horn area, immediately below the central channel, according to Grossman et al. ${ }^{20}$ This area was selected for the counting of neurons. 
model was used to compare the amplitudes and latencies between groups. Histopathological data for the site of injury was analyzed with the nonparametric Kruskal-Wallis test. The fiber counts distal/proximal to the lesion were analyzed with ANOVA.

\section{Treatment evaluations}

All evaluations were blinded, that is, the BBB scale tests (performed by GB and RMM), the evoked potential test (performed by RF), the pathological analysis (performed by RPO), and the statistical analysis.

\section{RESULTS}

Two rats died in each group ( $16.7 \%$ of the total sample); thus, a total of 40 animals survived to the $42 \mathrm{nd}$ day, and these were included in the final analyses. The causes of death were: (Group 1) two rats from urinary infection in the second week; (Group 2) one rat from infection in the second week, one from autophagia in the third week; (Group 3) two rats from autophagia in the second week; (Group 4) two rats from autophagia, one in the second week and one in the third week. The Dixon test did not identify any outliers to be removed from the sample.

The Student's $t$-test showed that the BBB scores were equal in limbs of the same rat. This allowed inclusion of each paw as an individual in the statistical analysis. For the evoked potential, front and hind limbs were considered individually.

The ANOVA showed significantly different BBB scores $(P \leqslant 0.002)$ from the 14th to the 42 nd day. Group 3, which received both antidepressant and physical therapy, had significantly higher BBB scores than the other three groups (Figure $2 ; P \leqslant 0.003$ ).

The evoked potential test showed that the hind limbs behaved differently between groups $(P \leqslant 0.003)$. The post hoc test pointed to a different behavior in Group 4 (controls). In the control group, latency was significantly higher $(P \leqslant 0.029)$ and amplitude was significantly lower $(P \leqslant 0.016)$ than in the other groups; this indicated that

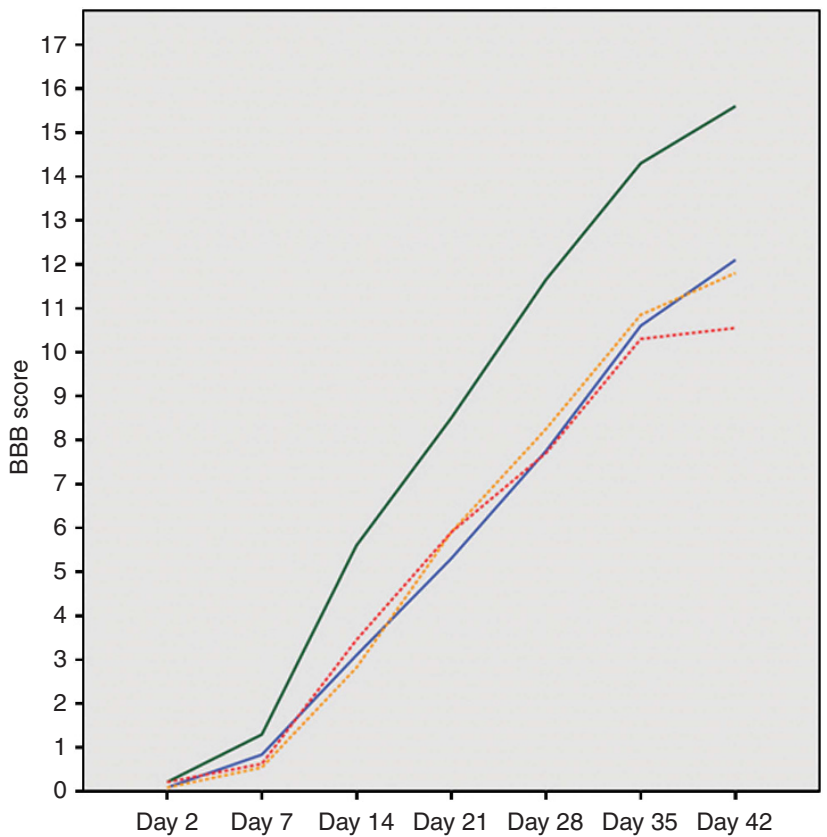

Figure 2 Average scores in the BBB scale according to study groups: blue line shows the group of rats trained in the treadmill, orange line shows the group receiving antidepressant, green line shows the combination treatment group and red line brings control group results.
Table 1 Multiple comparisons between groups (Bonferroni test) for the evoked potential results in hind legs of Wistar rats

\begin{tabular}{|c|c|c|c|c|c|}
\hline $\begin{array}{l}\text { Dependent } \\
\text { variable }\end{array}$ & $\begin{array}{c}\text { (I) } \\
\text { group }\end{array}$ & $\begin{array}{c}(J) \\
\text { group }\end{array}$ & $\begin{array}{c}\text { Mean difference } \\
(I-J)\end{array}$ & $\begin{array}{c}\text { Standard } \\
\text { error }\end{array}$ & $P$ \\
\hline \multicolumn{6}{|l|}{ Latency } \\
\hline & & 2 & -46.40 & 113.99 & 1.000 \\
\hline & 1 & 3 & -0.280 & 113.99 & 1.000 \\
\hline & & 4 & -377.90 & 113.99 & 0.008 \\
\hline & & 1 & 46.40 & 113.99 & 1.000 \\
\hline & 2 & 3 & 43.60 & 113.99 & 1.000 \\
\hline & & 4 & -331.50 & 113.99 & 0.029 \\
\hline & & 1 & 0.280 & 113.99 & 1.000 \\
\hline & 3 & 2 & -43.60 & 113.99 & 1.000 \\
\hline & & 4 & -375.10 & 113.99 & 0.009 \\
\hline & & 1 & 377.90 & 113.99 & 0.008 \\
\hline & 4 & 2 & 331.50 & 113.99 & 0.029 \\
\hline & & 3 & 375.10 & 113.99 & 0.009 \\
\hline \multicolumn{6}{|l|}{ Amplitude } \\
\hline & & 2 & 66.67 & 47.19 & 0.971 \\
\hline & 1 & 3 & 104.95 & 47.19 & 0.175 \\
\hline & & 4 & 251.58 & 47.19 & 0.000 \\
\hline & & 1 & -66.67 & 47.19 & 0.971 \\
\hline & 2 & 3 & 38.28 & 47.19 & 1.000 \\
\hline & & 4 & 184.91 & 47.19 & 0.001 \\
\hline & & 1 & -104.95 & 47.19 & 0.175 \\
\hline & 3 & 2 & -38.28 & 47.19 & 1.000 \\
\hline & & 4 & 146.63 & 47.19 & 0.016 \\
\hline & & 1 & -251.58 & 47.19 & 0.000 \\
\hline & 4 & 2 & -184.915 & 47.19 & 0.001 \\
\hline & & 3 & -146.635 & 47.19 & 0.016 \\
\hline
\end{tabular}

Significant values are in bold

${ }^{a}$ Group 1 received physical therapy; Group 2 received antidepressant; Group 3 received physical therapy plus antidepressant and Group 4 received no treatment (control).

untreated rats showed worse motor performance (Table 1) than those that received antidepressant, physical therapy, or both treatments (Table 2).

Hematoxylin-eosin staining showed no differences among groups for any variable $(P \geqslant 0.9)$. Fiber counts with electron microscopy also showed no differences among groups $(P=0.79)$. The ANOVA confirmed that the groups were not different in the number of regenerated axonal fibers (Figures 3 and 4).

\section{DISCUSSION}

No current treatment provides complete functional recovery after SCI. Available strategies include adaptive changes (plasticity) within the central nervous system and surgical repair. The present study showed that some functional recovery could occur without histologically evident changes in axonal fibers. Previous studies have also shown functional recovery without an obvious underlying anatomical change in the spinal cord lesion. ${ }^{13,21,22}$ The mechanisms that underlie our observations of increased $\mathrm{BBB}$ scores and evoked potential tests in 
Table 2 Descriptive values (mean, s.d., s.e.) for BBB scores in each group in each moment of observation

\begin{tabular}{|c|c|c|c|c|}
\hline & $\mathrm{N}$ & Mean & s.d. & s.e. \\
\hline \multicolumn{5}{|l|}{2} \\
\hline Lesion + treadmill & 24 & 0.08 & 0.282 & 0.058 \\
\hline Lesion + antidepressant & 24 & 0.08 & 0.282 & 0.058 \\
\hline Lesion + treadmill + antidepressant & 24 & 0.21 & 0.415 & 0.085 \\
\hline Lesion (control) & 24 & 0.21 & 0.509 & 0.104 \\
\hline Total & 96 & 0.15 & 0.383 & 0.039 \\
\hline \multicolumn{5}{|l|}{7} \\
\hline Lesion + treadmill & 24 & 0.83 & 1.341 & 0.274 \\
\hline Lesion + antidepressant & 24 & 0.54 & 0.833 & 0.170 \\
\hline Lesion + treadmill + antidepressant & 24 & 1.29 & 1.042 & 0.213 \\
\hline Lesion (control) & 24 & 0.63 & 0.824 & 0.168 \\
\hline Total & 96 & 0.82 & 1.056 & 0.108 \\
\hline \multicolumn{5}{|l|}{14} \\
\hline Lesion + treadmill & 20 & 3.10 & 2.292 & 0.512 \\
\hline Lesion + antidepressant & 22 & 2.82 & 0.958 & 0.204 \\
\hline Lesion + treadmill + antidepressant & 20 & 5.60 & 2.088 & 0.467 \\
\hline Lesion (control) & 20 & 3.45 & 1.986 & 0.444 \\
\hline Total & 82 & 3.72 & 2.150 & 0.237 \\
\hline \multicolumn{5}{|l|}{21} \\
\hline Lesion + treadmill & 20 & 5.30 & 2.105 & 0.471 \\
\hline Lesion + antidepressant & 20 & 5.90 & 2.573 & 0.575 \\
\hline Lesion + treadmill + antidepressant & 20 & 8.50 & 2.893 & 0.647 \\
\hline Lesion (control) & 20 & 5.90 & 3.259 & 0.729 \\
\hline Total & 80 & 6.40 & 2.962 & 0.331 \\
\hline \multicolumn{5}{|l|}{28} \\
\hline Lesion + treadmill & 20 & 7.75 & 1.943 & 0.435 \\
\hline Lesion + antidepressant & 20 & 8.25 & 2.613 & 0.584 \\
\hline Lesion + treadmill + antidepressant & 20 & 11.65 & 3.183 & 0.712 \\
\hline Lesion (control) & 20 & 7.70 & 2.755 & 0.616 \\
\hline Total & 80 & 8.84 & 3.087 & 0.345 \\
\hline \multicolumn{5}{|l|}{35} \\
\hline Lesion + treadmill & 20 & 10.60 & 2.624 & 0.587 \\
\hline Lesion + antidepressant & 20 & 10.85 & 2.477 & 0.554 \\
\hline Lesion + treadmill + antidepressant & 20 & 14.30 & 2.993 & 0.669 \\
\hline Lesion (control) & 20 & 10.30 & 2.342 & 0.524 \\
\hline Total & 80 & 11.51 & 3.044 & 0.340 \\
\hline \multicolumn{5}{|l|}{42} \\
\hline Lesion + treadmill & 20 & 12.10 & 2.614 & 0.584 \\
\hline Lesion + antidepressant & 20 & 11.80 & 2.546 & 0.569 \\
\hline Lesion + treadmill + antidepressant & 20 & 15.60 & 2.583 & 0.578 \\
\hline Lesion (control) & 20 & 10.55 & 1.905 & 0.426 \\
\hline Total & 80 & 12.51 & 3.040 & 0.340 \\
\hline
\end{tabular}

experimental rats without evidence of axonal regeneration remain to be discussed and might certainly inspire new research. We found that the combined treatment (exercise plus antidepressant) and the isolated treatments triggered different responses. This suggested that both chemistry and training can interfere with plasticity.

We used fluoxetine because it induces production of neurotrophic factors, like serotonin. ${ }^{11,23}$ Serotonin receptors are known to be involved in motor recovery and neurotrophic action. ${ }^{24}$ Fluoxetine is a

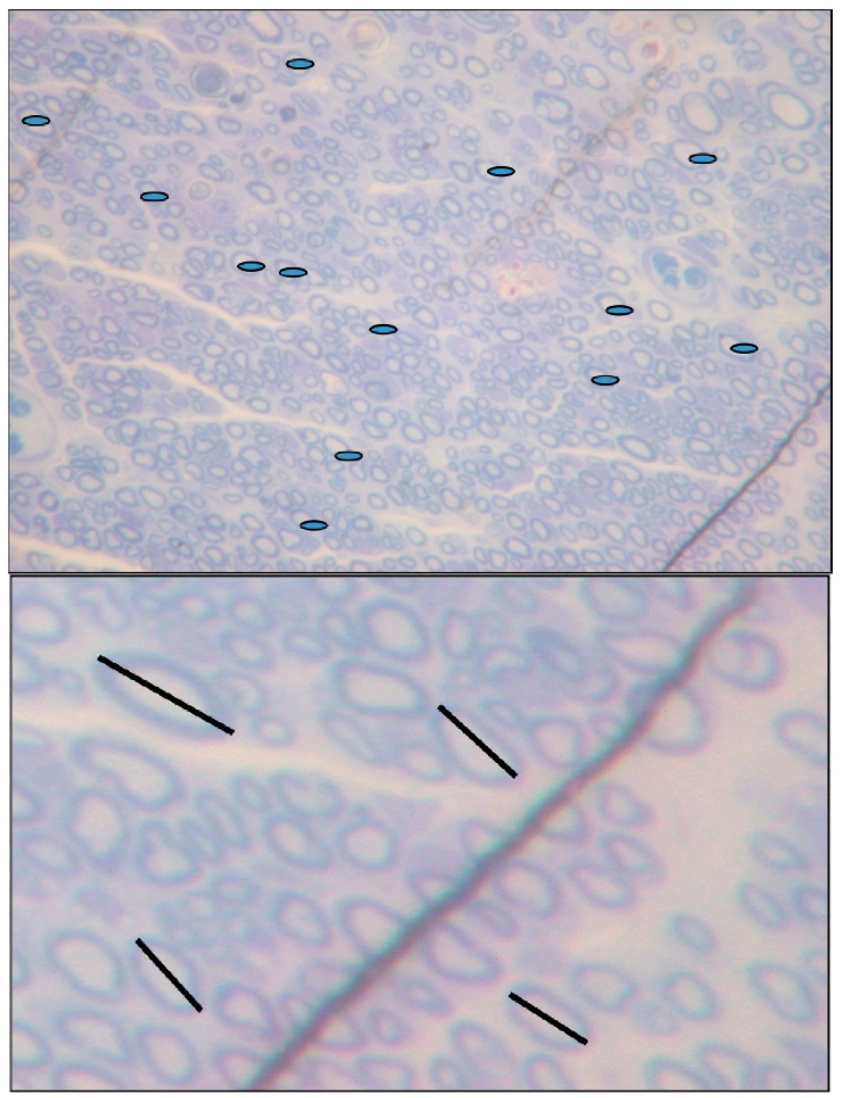

Figure 3 Above: fiber count in electronic microscopy; below: measurement of the fiber diameter (toluidine blue, $\times 10000$ ).

selective serotonin reuptake inhibitor, frequently used in patients living with spinal cord injuries who develop psychiatric diseases, particularly depression, in the chronic post-traumatic phase. ${ }^{25}$ Another neuroregenerative agent, lithium carbonate, was investigated, but it did not improve the neurological outcome in patients with SCI. ${ }^{26}$

Many studies have shown the effects of exercise training on neuronal plasticity. Exercise improved gait and led to neurological recovery, ${ }^{27}$ probably by enabling the intrinsic neuronal circuitry. ${ }^{28}$ The training regimen used in our study was based on a regimen previously described by Petruska et al. ${ }^{29}$ and Ferreira et al. ${ }^{30}$ Petruska et al. ${ }^{29}$ used $15-\mathrm{min}, 6-21 \mathrm{~cm} \mathrm{~s}^{-1}$ treadmill training with SpragueDawley rats that had undergone surgical transections of the spinal cords. Those rats showed significant changes in the cellular properties of motor neurons and the synaptic input from spinal white matter and muscle spindle afferents. Thus, training altered the electrophysiological parameters. Furthermore, the animals injured as neonates showed greater susceptibility to training; that finding suggested that the age at injury may influence plasticity. ${ }^{29}$ Ferreira et al. ${ }^{30}$ used short, daily training (like ours); they suggested that moderate physical exercise could modulate synaptic and structural proteins in motor brain areas, which may play an important role in exercise-dependent brain plasticity. ${ }^{30}$ This synergistic effect had been studied previously by Engesser-Cesar et al., ${ }^{11}$ who examined the effect of intraperitoneal fluoxetine and treadmill training on production of the hippocampal brain-derived neurotrophic factor and neurogenesis. That experimental model inspired us to test the synergistic action of combined treatments in functional evaluations. 

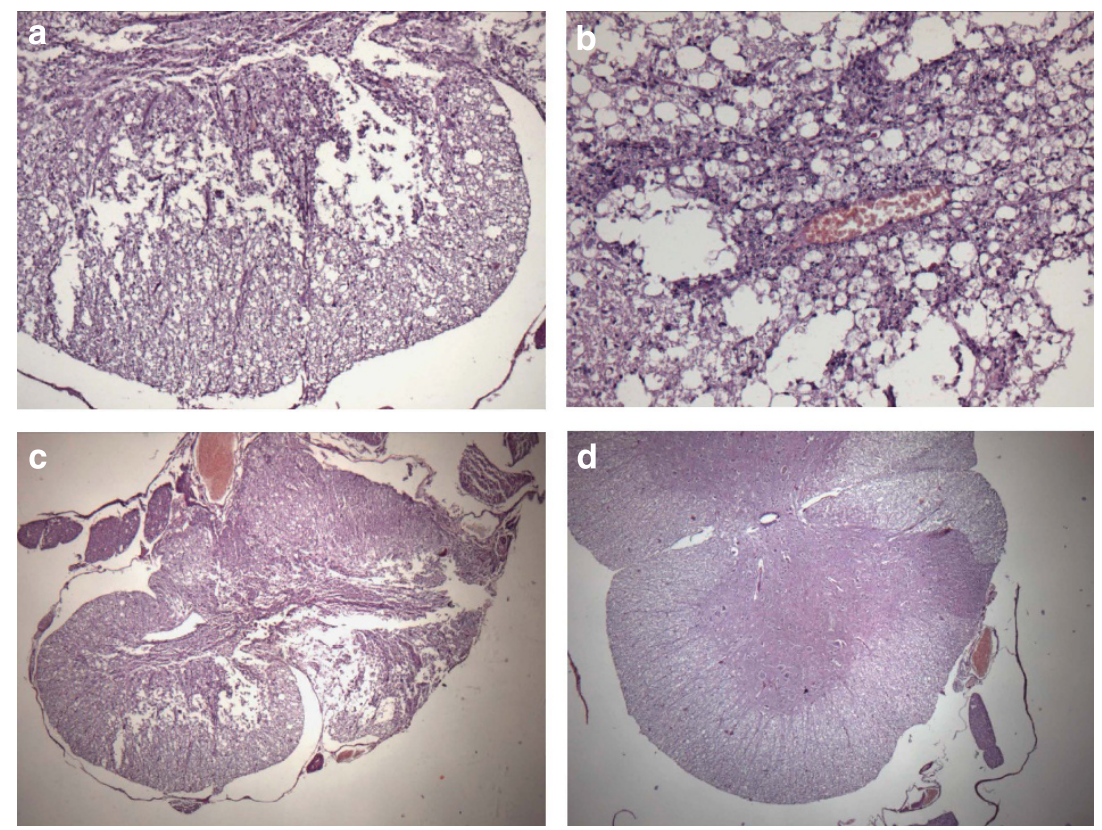

Figure 4 Histological evaluation with hematoxylin-eosin staining: (a) Intense degeneration of the nervous substance of the injured spinal cord tissue ( $\times 100)$; (b) marked degree of hyperemia $(\times 200)$; (c) Moderate cellular infiltrate and cystic degeneration $(\times 50)$, (d) region next to the lesion, without alterations $(\times 50)$.

The motor-evoked potential test showed that both the combination and isolated treatments provided improvement compared with no treatment. The amplitude of the motor-evoked potential represents the proportion of motor units that are recruited by the stimulus. When a supramaximum stimulus is delivered, as in this study, the amplitude can reveal differences in the behavior of muscle groups (there may be variation in the impulses, because the muscle units that are depolarized may vary for each stimulus). These differences in behavior could be due to neural regeneration or due to the strengthening of existing neural connections. Sometimes, neurophysiological changes precede histological changes, because structural changes can take a long time to develop. For example, neurophysiological changes are commonly detected in asymptomatic patients. ${ }^{13,21,22}$

In this study, the BBB scale evaluation showed that rats that received the combined treatment exhibited significantly better recovery than the other groups. This recovery occurred after the 14th day post-SCI. The groups treated with either antidepressant or treadmill training alone did not show statistically significant differences from the control group. The control group only showed clear differences from the others after day 35. Had the experiment continued, the difference between groups might have continued to grow, and eventually it might have reached significance. At 6 weeks, the control group was significantly different from the combined therapy group. A longer study should be conducted to determine whether a longer period of exercise alone would provide recovery of function, or whether the antidepressant is required to improve the training results. Alternatively, perhaps a longer follow-up would have shown a functional recovery in the BBB results, consistent with that evident in the evoked potential test.

We have used the same protocol for spinal cord lesion and the same device (the NYU Impactor) as in the studies by Basso et al. ${ }^{14}$ and Agrawal et al. ${ }^{31}$, and still we observed lower BBB values. The only difference between our study and theirs, which would explain the lower values, is the breed of the animals: they used Sprague-Dawley rats and we used Wistar rats. Sprague-Dawley rats are not easy to find in Brazilian laboratories, while Wistar rats are available at our university vivarium. This availability has allowed our research group to standardize procedures in spinal cord lesion studies, strictly according to the Multicenter Animal Spinal Cord Injury Study (MASCIS) standards, with many publications. Besides, the 48 rats in our study presented the same scoring up to the second week of observation, and this is a proof of the lesion standardization. Our NYU Impactor equipment is calibrated and the same score standards repeat in all other studies by our group.

The protocol for spinal cord lesion in this study was described in detail by Santos et al., ${ }^{13}$ based on MASCIS, and the lesion was produced using a $10-\mathrm{g}$ impact at a height of $12.5 \mathrm{~mm}$. The contusion model of SCI, performed using the NYU-MASCIS (New York University-Multicenter Animal Spinal Cord Injury Study) Impactor is a widely used clinically-relevant rodent model for experimental SCI research. The NYU-MASCIS weight-drop model standardizes grades of contusive SCI by dropping a $10 \mathrm{~g}$ rod from specific heights of 6.25 (mild), 12.5 (moderate), 25 (severe) or $50 \mathrm{~mm}$ (very severe) upon the exposed dorsal surface of the spinal cord. The NYU Impactor device tracks the trajectory of the falling $\operatorname{rod}( \pm 10 \mu \mathrm{m}$ and $\pm 10 \mu \mathrm{s})$ and measures impact velocity (Vi), cord compression distance (Cd), time $(\mathrm{Ct})$ and rate $(\mathrm{Cr}=\mathrm{Cd} / \mathrm{Ct})$. This computerized system allows the grade of the injury to be standardized. The NYU system monitors the effectiveness of the injury, the position of the rod, the speed curve for rod impact, the tangent curve of impact velocity, the exact point of impact, the maximum height, the inflection, the baseline, the vertebral position curve and the curve for the position of the vertebral impact. All these parameters are controlled, so that the researchers are sure that the lesion is always the same and effective. If by any reason the impact is different, the rat must be discarded. However, 
no rat in this study needed to be discarded for experimental errors such as these.

The MASCIS protocol was chosen because it allows the reproduction of the same experimental parameters by other research institutions. Other precautions were taken regarding the homogeneity of the study conditions: rigid inclusion criteria (only male rats, with ages between 20 and 21 weeks, and controlled weight, between 300 and $340 \mathrm{~g}$; only rats with normal motility and good health) provide reliability and reproducibility to the study.

Another possible reason for our lower values might be an interobserver variation during BBB scoring. BBB scores interobserver variation is higher in more severe lesions (exactly as seen in the first 2 weeks in our study). The BBB scale 20-22 is the main tool used to quantify the motor recovery in rats with spinal cord lesion, according to the MASCIS (Multicenter Animal Spinal Cord Injury Study) since 1996. It was adopted in our laboratory to easily evaluate spinal cord lesions in T VII, T VIII and T IX. The BBB scale provides a scoring between 0 and 21 to evaluate the functional, sensitive and motor responses, and it is very sensible to little modifications in the locomotor function of the rats. However, Metz et al. ${ }^{32}$ verified that the definitions and criteria for the determination of the motor capacity by the observers vary considerably. The authors argue that $\mathrm{BBB}$ should be combined to other tools in the evaluation of function after SCI, in order that the results from different laboratories can be compared. Ferguson et al. ${ }^{33}$ verified that the distribution of scores is irregular during BBB scoring: the scores 2 and 3 are rarely used. The authors state that the discontinuity can result in problems for statistical analyses, parametric or nonparametric. Therefore, one observer can easily be mistaken in his individual evaluation by the BBB score when facing severe spinal lesions.

In addition to functional and motor evaluations, this study verified the results of treatment with histopathological analyses. Histology was performed to detect signs of cellular plasticity after treatment for spinal cord lesions. The spinal tissue was evaluated in three segments: within the lesion area and outside the lesion, in the proximal and distal areas. However, the toluidine blue staining and electronic microscopy showed no difference between groups in axonal fiber counts. This was probably because we chose a moderate lesion in this study to show differences that would be evaluable by functional analysis.

Science is unraveling the mechanisms of cell protection and neuroregeneration; however, clinically, we can only provide supportive care for patients with SCI. This combination treatment may represent an alternative that enhances functional recovery of patients with SCIs.

\section{CONCLUSIONS}

The combination of gait training and antidepressant treatment with fluoxetine significantly improved $\mathrm{BBB}$ scores compared with no treatment or physical therapy or antidepressant alone. These changes occurred from 14 to 42 days after the SCI, but were not evident in the histological analysis. However, in the motor-evoked potentials evaluation, all three experimental groups showed superior neurological recovery compared with the control group.

\section{DATA ARCHIVING}

There were no data to deposit.

\section{CONFLICT OF INTEREST}

The authors declare no conflict of interest.
1 van Middendorp JJ, Barbagallo G, Schuetz M, Hosman AJ. Design and rationale of a Prospective, Observational European Multicenter study on the efficacy of acute surgical decompression after traumatic Spinal Cord Injury: the SCI-POEM study. Spinal Cord 2012; 50, 686-694.

2 Foret A, Quertainmont R, Botman O, Bouhy D, Amabili P, Brook G et al. Stem cells in the adult rat spinal cord: plasticity after injury and treadmill training exercise. $J$ Neurochem 2010; 112, 762-772.

3 Liu M, Bose P, Walter GA, Thompson FJ, Vandenborne K. A longitudinal study of skeletal muscle following spinal cord injury and locomotor training. Spinal Cord 2008; 46, 488-493.

4 Schwab ME, Bartholdi D. Degeneration and regeneration of axons in the lesioned spinal cord. Physiol Rev 1996; 76, 319-370.

5 Blight AR, Zimber MP. Acute spinal cord injury: pharmacotherapy and drug development perspectives. Curr Opin Investig Drugs 2001; 2, 801-808.

6 Ung RV, Landry ES, Rouleau P, Lapointe NP, Rouillard C, Guertin PA. Role of spinal 5$\mathrm{HT} 2$ receptor subtypes in quipazine-induced hindlimb movements after a low-thoracic spinal cord transaction. Eur J Neurosci 2008; 28, 2231-2242.

7 Gerin CG, Hill A, Hill S, Smith K, Privat A. Serotonin release variations during recovery of motor function after a spinal cord injury in rats. Synapse 2010;64, 855-861.

8 Kao T, Shumsky JS, Jacob-Vadakot S, Himes BT, Murray M, Moxon KA. Role of the 5$\mathrm{HT} 2 \mathrm{C}$ receptor in improving weight-supported stepping in adult rats spinalized as neonates. Brain Res 2006; 1112, 159-168.

9 Hayashi Y, Jacob-Vadakot S, Dugan EA, McBride S, Olexa R, Simansky K et al. 5-HT precursor loading, but not 5-HT receptor agonists, increases motor function after spinal cord contusion in adult rats. Exp Neurol 2010; 221, 68-78.

10 Ichiyama RM, Gerasimenko Y, Jindrich DL, Zhong H, Roy RR, Edgerton VR. Dose dependence of the $5-\mathrm{HT}$ agonist quipazine in facilitating spinal stepping in the rat with epidural stimulation. Neurosci Lett 2008; 438, 281-285.

11 Engesser-Cesar C, Anderson AJ, Cotman CW. Wheel running and fluoxetine antidepressant treatment have differential effects in the hippocampus and the spinal cord. Neuroscience 2007; 144, 1033-1044.

12 Cristante AF, Damasceno ML, Barros Filho TE, de Oliveira RP, Marcon RM, da Rocha ID. Evaluation of the effects of hyperbaric oxygen therapy for spina cord lesion in correlation with the moment of intervention. Spinal Cord 2012; 50, 502-506.

13 Santos GB, Cristante AF, Marcon RM, Souza FI, Barros Filho TEP, Damasceno ML. Modelo experimental de lesão medular e protocolo de avaliação motora em ratos wista (Spinal cord injury experimental model and motion evaluation protocol in wistar rats). Acta Ortop Bras 2011; 19, 87-91.

14 Basso DM, Beattie MS, Bresnahan JC, Anderson DK, Faden AI, Gruner JA et al MASCIS evaluation of open field locomotor scores: effects of experience and teamwork on reliability. Multicenter Animal Spinal Cord Injury Study. J Neurotrauma 1996; 13, 343-359.

15 Moyses ZP, Nakandakari FK, Magaldi AJ. Fluoxetine effect on kidney water reabsorption. Nephrol Dial Transplant 2008; 23, 1173-1178.

16 Basso DM, Beattie MS, Bresnahan JC. A sensitive and reliable locomotor rating scale for open field testing in rats. J Neurotrauma 1995; 12, 1-21.

17 Souza FI, Barros Filho TEP, Cristante AF. Avaliação do emprego do GM1 após lesão medular experimental em ratos (Evaluation of the use of GM1 after experimental spinal cord injury in rats). Coluna/Columna 2011; 10, 305-308.

18 Chopek JW, Gardiner PF. Life-long caloric restriction: effect on age-related changes in motoneuron numbers, sizes and apoptotic markers. Mech Ageing Dev 2010; 131, $650-659$

19 Bertelli JA, dos Santos AR, Taleb M, Calixto JB, Mira JC, Ghizoni MF. Long interpositional nerve graft consistently induces incomplete motor and sensory recovery in the rat. An experimental model to test nerve repair. J Neurosci Methods 2004; 134, 75-80.

20 Grossman RG. Cell transplantation in Parkinson's disease: implications for human clinical trials. Neurosurgery 2001; 49, 580-582.

21 Cristante AF, Barros Filho TE, Oliveira RP, Marcon RM, Rocha ID, Hanania FR et al. Antioxidative therapy in contusion spinal cord injury. Spinal Cord 2009; 47, 458-463.

22 Marcon RM, Cristante AF, de Barros Filho TE, de Oliveira RP, dos Santos GB. Potentializing the effects of GM1 by hyperbaric oxygen therapy in acute experimental spinal cord lesion in rats. Spinal Cord 2010; 48, 808-813.

23 Fouad K, Rank MM, Vavrek R, Murray KC, Sanelli L, Bennett DJ. Locomotion after spinal cord injury depends on constitutive activity in serotonin receptors. J Neurophysiol 2010; 104, 2975-2984.

24 Bharne AP, Upadhya MA, Kokare DM, Subhedar NK. Effect of alpha-melanocyte stimulating hormone on locomotor recovery following spinal cord injury in mice: role of serotonergic system. Neuropeptides 2011; 45, 25-31.

25 Kemp BJ, Kahan JS, Krause JS, Adkins RH, Nava G. Treatment of major depression in individuals with spinal cord injury. J Spinal Cord Med 2004; 27, 22-28.

26 Yang ML, Li JJ, So KF, Chen JY, Cheng WS, Wu J et al. Efficacy and safety of lithium carbonate treatment of chronic spinal cord injuries: a double-blind, randomized, placebo-controlled clinical trial. Spinal Cord 2012; 50, 141-146.

27 Holschneider DP, Yang J, Guo Y, Maarek JM. Reorganization of functional brain maps after exercise training: Importance of cerebellar-thalamic-cortical pathway. Brain Res 2007; 1184, 96-107.

28 Ferreira R, Oliveira AR, Barros Filho TEP. Padronização da técnica para captação do potencial evocado motor em ratos através da estimulação elétrica transcraniana 
(Standardization of motor evoked potential captivation technique in rats through transcranial electric stimulus). Acta Ortop Bras 2005; 13, 112-114.

29 Petruska JC, Ichiyama RM, Jindrich DL, Crown ED, Tansey KE, Roy RR et al. Changes in motoneuron properties and synaptic inputs related to step training after spinal cord transection in rats. J Neurosci 2007; 27, 4460-4471.

30 Ferreira AF, Real CC, Rodrigues AC, Alves AS, Britto LR. Moderate exercise changes synaptic and cytoskeletal proteins in motor regions of the rat brain. Brain Res 2010; $1361,31-42$.
31 Agrawal G, Kerr C, Thakor NV, All AH. Characterization of graded multicenter animal spinal cord injury study contusion spinal cord injury using somatosensory-evoked potentials. Spine (Phila Pa 1976) 2010; 35, 1122-1127.

32 Metz GA, Merkler D, Dietz V, Schwab ME, Fouad K. Efficient testing of motor function in spinal cord injured rats. Brain Res 2000; 883, 165-177.

33 Ferguson AR, Hook MA, Garcia G, Bresnahan JC, Beattie MS, Grau JW. A simple post hoc transformation that improves the metric properties of the BBB scale for rats with moderate to severe spinal cord injury. J Neurotrauma 2004; 21, 1601-1613. 